\title{
NETWORK PLANNING AND CAPACITY MANAGEMENT CONSIDERING ADAPTIVE SECTORIZATION IN SURVIVABLE FDMA/CDMA SYSTEMS
}

\author{
Kuo-Chung Chu ${ }^{\dagger, \ddagger}$ and Frank Yeong-Sung Lin ${ }^{\dagger}$ \\ ${ }^{\dagger}$ Department of Information Management, National Taiwan University \\ ${ }^{\ddagger}$ Department of Information Management, Jin-Wen Institute of Technology \\ Taipei, Taiwan
}

\begin{abstract}
In this paper, we investigate the integrated problem of survivable CDMA network planning and management. The problem is formulated as a combinatorial optimization formulation in terms of deploying cost minimization. Solution approach to the development algorithm is Lagrangean relaxation. In experiment results, the proposed algorithm is shown to be more effective and achieves up to $25 \%$ reduction on the total deployment cost over a simple algorithm. It also takes around $30 \%$ expenses to guarantee survivability.
\end{abstract}

\section{INTRODUCTION}

Generally, the issues of network planning consist of allocation for mobile telephone switching offices (MTSO), base stations (BS), backbone topology, and system configuration. Since backbone topology is a key factor in the routing problem, the backbone topology and the routing path for each O-D (origination-destination) pair must be jointly considered. Unlike secondgeneration cellular networks, CDMA system capacity bounded on interferences in both uplink and downlink is another issue of system planning.

To mitigate the interferences in terms of capacity maximization, sectorization is an effective way [1][2]. Denote $K$ the set of sector configurations and $S$ the set of sector candidates, sector candidate $s_{k, i}$ is defined by both sector configuration $(k \in K)$ and sector identity (i). For simplicity, $s_{k, i}$ is substituted by $s$, and denote sector $_{j s}$ the sector $s$ in BS $j\left(\forall s \in S_{k}, j \in B\right)$, where $B$ is the set of base stations. Without loss of generality, the interferences between users can be treated as the interferences between sectors. Besides, combining traditional FDMA to CDMA is an alternative to moderately mitigate interferences. [3] shows that the current frequency reuse factor of 1 is not always optimal. Another study demonstrated that it is possible to increase the cell capacity without deploying additional cells by applying proper FDMA frequency reuse to minimize interference [4].

Although intensive research on different issues of network planning has been conducted, relatively little work attempting to deal with the overall planning and management problem in an integrated and joint manner has been seen in the literature. Previous studies of CDMA planning focused on cell arrangement [5][6]. Chu et al. [7] first developed a mathematical model to the aforementioned overall planning and management problem. However, sectorization and spectrum resource allocation were not considered in their work. In this paper, we investigate CDMA network planning which jointly considers adaptive sectorization and hybrid F/CDMA scheme under QoS/GoS and survivability constraints. The remainder of this paper is organized as follows. In Section 2, hybrid F/CDMA scheme is introduced. Section 3 presents the integrated model of network planning as well as solution approach. Section 4 illustrates the computational experiments. Finally, Section 5 concludes this paper.

\section{HYBRID F/CDMA SCHEME}

In hybrid F/CDMA scheme, the available wideband spectrum is divided into a number of sub-bands with smaller bandwidths. Each sub-band employs direct sequence (DS) spreading with reduced processing gain and is transmitted in one and only one sub-band. The capacity of this F/CDMA system is calculated as the sum of the capacities of the sub-band. Given the whole bandwidth $B W_{\text {WHOLE }} 60 \mathrm{MHZ}$ in both uplink (UL) and downlink (DL), which is made up of $N_{F U}$ frequency units (FU) with $B W_{F U}=6 \mathrm{MHZ}$, where $N_{F U}=B W_{W H O L E} / B W_{F U}=10$. Denote $M$ the set of sub-bands in length of frequency segment (FS). By integrating FU, a number of FS in both UL (denote $W_{j s}^{U L}$ ) and DL (denote $W_{j s}^{D L}$ ), so-called sub-band, can be separated. FS instead of whole bandwidth (reuse factor of 1) is deployed in sector $_{j s}$. The term frequency segment (FS) and sub-band will be used in turn throughout the paper.

Given sector configuration, the interference indicator functions $\Omega_{j s j^{\prime} s^{\prime}}^{U L}$ and $\Omega_{j s j^{\prime} s^{\prime}}^{D L}$ for $\mathrm{UL}$ and DL from sector $_{j s}$ to sector $_{j^{\prime} s^{\prime}}$, respectively, can be pre-calculated. Let $y_{j s m}$ be the decision variable which is 1 if sector $_{j s}$ deploys $m$-th sub-band in both $U L$ and $D L$, or 0 otherwise. Thus, bandwidth allocated for uplink 
and downlink is calculated by $\mathrm{W}_{j s}^{U I}=\sum_{m \in M} y_{j s m} \cdot L_{m} \cdot B W_{F V}^{U L}$ and $\mathrm{W}_{j s}^{D L}=\sum_{m \in M} y_{j s m} \cdot L_{m} \cdot B W_{F U}^{D L}$, respectively, where $L_{u}$ is the length of $m$-th sub-band. Sub-bands deployed in any two sector $_{j s}$ probably exist overlapping of FU. Furthermore, the interference indicator function $\Psi_{m m^{\prime}}^{U / L}$. ( $\Psi_{m m^{\prime}}^{D L}$ ) from $m$ to $m^{\prime}$ can be pre-calculated.

For traffic distribution, denote $C$ the set of traffic classes and $c(o)(c(o) \in C)$ the traffic class of OD pair $o(\forall o \in O)$, where $O$ is the set of OD pair. If traffic type of OD $o$ belongs to class- $c$, class- $c(o)$ is equivalent to class- $c$. Let $d_{c(t)}^{U L}\left(d_{c(t)}^{D L}\right)$ be the information rate in uplink (downlink): Denote $z_{j s t}$ the decision variable which is 1 if MS $t$ is granted by sector $_{j s}$ or 0 otherwise. Assuming both link powers are perfectly. controlled, it ensures the received power at sector $_{i s}$ from MS $t$ with constant value in same traffic class- $c(t)$. Denote $P_{c(t)}^{U L}\left(P_{c(t)}^{D L}\right)$ the received uplink (downlink) power signal, the uplink signal-to-interference ratio (SIR) $S I R_{j+,(t)}^{v r}=$

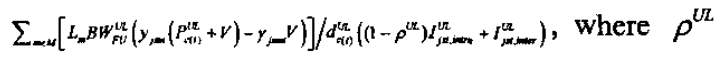
is the uplink orthogonality factor, $\alpha_{c(t)}^{U L}$ is uplink activity factor. A very large constant value $V$ in numerator is to satisfy constraint requirement if MS $t$ is rejected $\left(z_{j s t}=0\right)$. Downlink is similar to uplink, given

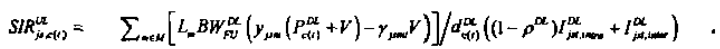
Since we jointly considers effect of inter-sector and inter-FS on inter-cell interference, the coupling of decision variables $\left(y_{j s m}, z_{j s t}\right)$ and decision variables $\left(y_{j^{\prime} s^{\prime} m^{\prime}}, y_{j s m}, z_{j s s}\right)$ results to non-linear form. Here we introduce auxiliary variables $\gamma_{j s m t}=y_{j s m} z_{j s t} \quad$ (s.t. $y_{j s m}+z_{j s t} \geq 2 \cdot \gamma_{j s m t}, \quad y_{j s m}+z_{j s t}-1 \leq \gamma_{j s m t} \quad$ and $\zeta_{j^{\prime} s^{\prime} u^{\prime} j \text { sut' }}=\gamma_{j^{\prime} s^{\prime} u^{\prime} t^{\prime}} y_{j s u}=y_{j^{\prime} s^{\prime} u^{\prime}}, z_{j^{\prime} s^{\prime} t^{\prime}} y_{j s u}$ (s.t. $\left.\gamma_{j^{\prime} s^{\prime} u^{\prime} t^{\prime}}+y_{j s u} \geq 2 \cdot \zeta_{j^{\prime} s^{\prime} u^{\prime} t^{\prime} j s u}, \quad \gamma_{j^{\prime} s^{\prime} u^{\prime} t^{\prime}}+y_{j s u}-1 \leq \zeta_{j^{\prime} s^{\prime} u^{\prime} t^{\prime} j s u}\right)$ so that non-linear form can be reduced to linear form. The detail definition is referred to [8].

\section{SYSTEM MODEL}

\subsection{Mathematical modeling}

To better describe the problem, the following subproblems are jointly investigated: (i) base station allocation subproblem; (ii) routing, network topology, and capacity management subproblem; (iii) mobile station homing and channel assignment subproblem; (iv) power transmission radius control subproblem; (v) bandwidth allocation subproblem. Denote $L_{1}, L_{2}, L_{3}$ the set of links between two MTSO, between MTSO and base station, between base station and mobile station, respectively. Given $L=L_{1} \cup L_{2} \cup L_{3}$. If the costs of base station installation, sector configuration, bandwidth allocation, and link connection are given $\Delta_{B}, \Delta_{k}, \Delta_{m}$, and $\Delta_{l}\left(c_{l}\right)$ with capacity $c_{l}$, respectively, the objective is to minimize total costs in (IP).

$$
\begin{aligned}
& Z_{I P}=\min \\
& \sum_{j \in B} \Delta_{B} a_{j}+\sum_{j \in B} \sum_{k \in X} \Delta_{k} b_{j k}+\sum_{j \in B} \sum_{s \in S} \sum_{m \in M} \Delta_{m} d_{j s m}+\sum_{j \in L} \Delta_{l}\left(c_{t}\right) \\
& \left(\frac{E_{\mathrm{b}}}{N_{\text {TOTN }}}\right)_{(t)}^{u} \leq S I R_{j s,(t)}^{(L)} \quad \forall j \in B k \in K, s \in \mathcal{S}_{k}, t \in T, e \in E(1) \\
& \left(\frac{E_{b}}{N_{\text {JOTAL }}}\right)_{c(t)}^{D L} \leq S I R_{j, c(t)}^{D L} \quad \forall \in B k \in K_{,} S \in S_{k}, t \in T, e \in E(2) \\
& \sum_{i \in L_{3}} \sum_{o \in O} \sum_{p \in P_{o}} A_{c(o)} x_{p}^{e} \delta_{p l} \phi_{i j s}=g_{j s c}^{e} \quad \forall \in \in B k \in K, s \in \bigwedge_{,}, c \in C, e \in E(3) \\
& \sum_{l \in L_{0}} \sum_{o \in O} \sum_{p \in \rho_{e}} q_{c(o)} x_{p}^{e} \delta_{p l} \phi_{l s}=q_{j s c}^{e} \quad \forall \in R, k \in K, s \in\left\{_{k}, c \in C, e \in E(4)\right. \\
& \sum_{p \in O} \sum_{p \in P_{o}} A_{c(o)} x_{p}^{e} \delta_{p l}=f_{l c} \quad \forall l \in L-\left\{L_{3}\right\}, c \in C, e \in E(5) \\
& \sum_{o \in O} \sum_{p \in P_{o}} q_{c(o)} x_{p}^{e} \delta_{p t}=n_{l c} \quad \forall l \in L-\left\{L_{3}\right\}, c \in C, e \in E(6) \\
& S B_{j s c}\left(g_{j s c}^{e}, m_{j s c}^{e}\right) \leq \beta_{j s c}^{e} \quad \forall j \in B, k \in K, s \in S_{k}, c \in C, e \in E(7) \\
& L B_{l c}\left(f_{l c}, n_{l c}\right) \leq \varepsilon_{l_{c}} \quad \forall l \in L-\left\{L_{3}\right\}, c \in C, e \in E(8) \\
& \sum_{p \in P_{O}} x_{p}^{e}=1 \quad \forall o \in O, e \in E(9) \\
& \sum_{o \in O} \sum_{p \in P_{p}} x_{p}^{e} \delta_{p l} \phi_{l j s} \rho_{l t} \leq z_{j s t}^{e} \quad \forall j \in B, k \in K, s \in S_{k}, l \in L_{s,}, t \in T, e \in E(10) \\
& z_{j s t}^{e} D_{j t} \leq r_{j s}^{e} \mu_{j s l}^{e} \quad \forall j \in B, k \in K, s \in S_{k}, t \in T, e \in E(11) \\
& 0 \leq r_{j s}^{e} \leq R_{j s} \quad \forall j \in B, k \in K, s \in S_{k}, r_{j s}^{e} \in Y, e \in E(12) \\
& b_{j k}^{e} \leq a_{j} \quad \forall j \in B, k \in K, e \in E(13) \\
& d_{j s m}^{e} \leq a_{j} \quad \forall j \in B, k \in K, s \in S_{k}, e \in E(14) \\
& \sum_{j \in B} \sum_{k \in K} \sum_{s \in S_{k}} z_{j s t}^{e}=1 \quad \forall t \in T, e \in E(15) \\
& \sum_{k \in K} b_{j k}^{e}=1 \quad \forall j \in B, e \in E(16) \\
& \sum_{m \in M} d_{j s m}^{e}=1 \quad \forall j \in B, k \in K, s \in S_{k}, e \in E(17) \\
& z_{j s t}^{e} \leq a_{j} \quad \forall j \in B, k \in K, s \in S_{k}, t \in T, e \in E(18) \\
& 2 \cdot \gamma_{j s m t}^{e} \leq y_{j s m}^{e}+z_{j s t}^{e} \quad \forall j, \in B, k \in K, s \in S_{k}, t \in T, m \in M(19) \\
& y_{j s m}^{e}+z_{j s t}^{e}-1 \leq \gamma_{j s m t}^{e} \quad \forall j, \in B, k \in K, s \in S_{k}, t \in T, m \in M(20)
\end{aligned}
$$

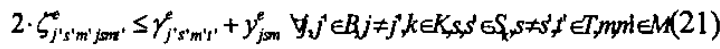

$$
\begin{aligned}
& \gamma_{j^{\prime} s^{\prime} m^{\prime} r^{\prime}}^{e}+y_{j o m}^{e}-1 \leq \zeta_{j^{\prime} s^{\prime} m^{\prime} j s m m^{\prime}}^{e} \forall, j^{\prime} \in R j \neq j^{\prime}, k \in K, s, s^{\prime} \in S_{k}, s \neq s^{\prime} f^{\prime} \in T, m m l \in M(22) \\
& a_{j}=0 \text { or } 1 \\
& \forall j \in B(23) \\
& b_{j k}^{e}=0 \text { or } 1 \\
& \forall j \in B, k \in K, e \in E(24) \\
& d_{j s m}^{e}=0 \text { or } 1 \quad \forall j \in B, k \in K, s \in S_{k}, m \in M, e \in E(25) \\
& x_{p}^{e}=0 \text { or } 1 \quad \forall 0 \in O, p \in P_{o}, e \in E(26) \\
& z_{j s t}^{e}=0 \text { or } 1 \quad \forall j \in B, k \in K, s \in S_{k}, t \in T, e \in E(27) \\
& y_{j s m}^{e}=0 \text { or } 1 \forall j, \in B, k \in K, s \in S_{k}, t \in T, m \in M, e \in E(28) \\
& \gamma_{j s m t}^{e}=0 \text { or } 1 \forall j, \in B, k \in K, s \in S_{k}, t \in T, m \in M, e \in E(29) \\
& \zeta_{j^{\prime} s^{\prime} m^{\prime} j s m t^{*}}=0 \text { or } 1 \\
& \forall j, j^{\prime} \in B, j \neq j^{\prime}, k \in K, s, s^{\prime} \in S_{k}, s \neq s^{\prime}, t^{\prime} \in T, m, m \in M(30)
\end{aligned}
$$


Denote $E$ the set of network state. SIR constraint for uplink and downlink is (1) and (2), respectively, in state $e \in E$. Denote $\delta_{p l}$ the indicator function which is 1 if link $l$ belongs to path $\mathrm{p}$ and 0 otherwise, and denote $\phi_{l j s}$ the indicator function which is 1 if sector $s$ in BS $j$ is one end of link $l$ and 0 otherwise. Given traffic intensity $A_{o}$ of $\mathrm{OD}$ pair $o \in O$ and routing decision variable $x_{p}^{e}$, which is 1 if path $p$ is selected at state $e$, the aggregate traffic (in Erlangs) in sector ${ }_{j s}$ and in link $l$ is given $g_{j s}^{e}=\sum_{c \in C} g_{j s c}^{e}$ and $f_{l}=\sum_{c \in C} f_{l c}$, where $g_{j s c}^{e}$ and $f_{l c}$ is the aggregate intensity of class- $c$ defined in (3) and (5). Denote $q_{j s}=\sum_{c \epsilon C} q_{j s c}\left(n_{l}=\sum_{c \in C} n_{l c}\right)$ the number of total channels allocated in sector ${ }_{j s}$ (link $l$ ), where $q_{j s c}$ and $n_{l}$ is the number of channels required for traffic class- $c$ defined in (4) and (6). Constraint (7) and (8) requires that a blocking in sector and link is under predefined threshold ( $\beta_{j s c}^{i}$ and $\varepsilon_{k c}$ ) of probability, respectively, where sector blocking $S B(\cdot)$ and link blocking $L B(\cdot)$ is the model developed by Kaufman [9]. Constraint (9) ensures that each O-D pair would be transmitted on one path. Constraint (10) is to guarantee that if a base station does not provide service to a mobile station, the link between them cannot be selected as a part of routing path.

Denote $\mu_{j s t}^{e}$ indication function is 1 if MS $t$ is covered by sector $s$ of MS $j$ at state $e$ or 0 otherwise. MS can be serviced in the coverage of BS by (11), where $r_{j s}^{e}$ and $D_{j t}$ is the power transmission radius and the distance to MS. Constraint (12) is to ensure that the transmission radius of each base station ranges from 0 to $R_{j}$. The following constraints are associated with bandwidth allocation. Constraint (13) ensures that sectorization is not deployed without base station installation, while (14) requires that the bandwidth is assigned only if the base station is installed. Constraint (15) guarantees that each mobile station is serviced. Constraint (16) requires only one sector configuration is deployed for each base station. A sector in any network state is allocated by one bandwidth in (17). If base station is not installed, no mobile station can be serviced by (18). Since two auxiliary decision variables are introduced, a number of constraints are listed from (19) to (22). (23)-(30) are integer properties of the decision variables.

\subsection{Solution approach}

To solving the complicated mathematical optimization model, Lagrangean relaxation method is applied [10]. Problem (IP) is transferred to be a dual problem by relaxing nine complicating constraints $(1)(2)(10)(11)(13)$ $(14)(18)(19)(20)$, then multiply the relaxed constraints with corresponding Lagrangean multipliers vector $V=\left(v_{1}\right.$, $\left.v_{2}, v_{3}, v_{4}, v_{5}, v_{6}, v_{7}, v_{8}, v_{9}\right)$ and add them to the primal objective function. We get Lagrangean relaxation (LR) problem, which is further decomposed into five independent subproblems with respect to decision variables. All of them can be optimally solved efficiently by proposed algorithms ' ${ }^{1}$. According to the weak Lagrangean duality theorem, for any $V \geq 0$, the objective value of $Z_{D}(V)$ is a lower bound of $Z_{I P}$. Thus, the dual problem $Z_{D}=\max Z_{D}(V)$ is constructed to calculate the tightest lower bound by adjusting multipliers subject to $V \geq 0$. Then, subgradient method is used to solving the dual problem. Let the vector $S$ is a subgradient of $Z_{D}(V)$ at $V \geq 0$. In iteration $k$ of subgradient optimization procedure, the multiplier vector $\pi$ is updated by $\pi^{k+1}=\pi^{k}+t^{k} S^{k}$, in which $t^{k}$ is a step size determined by $t^{k}=\lambda\left(Z_{I P}^{*}-Z_{D}\left(\pi^{k}\right)\right) /\left\|S^{k}\right\|^{2}$, where $Z_{I P}^{*}$ is an upper bound on the primal objective function value after iteration $k$, and $\lambda$ is a constant where $0 \leq \lambda \leq 2$. To calculate upper bound of (IP), the algorithm of getting primal feasible solutions is also proposed ${ }^{2}$. In order to prove the efficiency of proposed Lagrangean relaxation (LR) algorithms, a simple algorithm ${ }^{3}(\mathrm{SA})$ is also implemented.

\section{EXPERIMENT RESULTS}

In this work, we consider voice $(v)$ and data $(d)$ traffics, $v, d \in C$. Number of OD pairs is half of total number of MS $(|O|=|T| / 2)$. Each OD pair belongs to only one traffic type, either voice or data, and the number of voice-data traffics is $50-50$ in all OD pairs. The traffic distribution among OD pairs is randomly generated. The experiment environment is given $|B|=15,|K|=2$ (omni-directional antenna and three uniform sectors). Associated GoS/QoS parameters are given $\varepsilon_{l v}=0.01$, $\varepsilon_{l d}=\beta_{j s v}^{e}=0.03, \beta_{j s d}^{e}=0.05$, the required bit energy-tonoise density for voice $(v)$ and data $(d)$ traffics

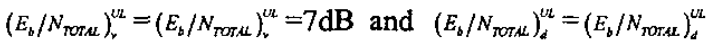
$=10 \mathrm{~dB}$, respectively. Activity factor of $\alpha_{\nu}$ and $\alpha_{d}$ in both links are all given 0.5 . Assigning the unit costs $\Delta_{B}=5,000,000, \quad \Delta_{k}=800,000, \quad \Delta_{m}=200,000$, and $\Delta_{l}=50$ per channel. Information rate $d_{v}^{U L}=d_{v}^{D L}=9.6 \mathrm{bps}$, $d_{d}^{U L}=d_{d}^{D L}=38.4 \mathrm{bps}$. Number of channel required $q_{v}=1$, $q_{d}=4$. Orthogonality factor $\rho^{v L}=0.9, \rho^{D L}=0.7$. Power is perfectly controlled by $P_{v}^{U L}=10 \mathrm{~dB}, P_{v}^{D L}=15 \mathrm{~dB}$, $P_{d}^{U L}=15 \mathrm{~dB}, P_{d}^{D L}=20 \mathrm{~dB}$.

To evaluate the performance of survivability issue, network states of both normal case ( $\mathrm{N}$-no base station failure) and failure case (F) are taken into account. All experiments are coded in $\mathrm{C}++$ and running on INTELTM P4-1.6GHZ CPU with $256 \mathrm{MB}$ RAM. For

\footnotetext{
1.2.3 Detailed algorithms are omitted due to the length limitation of the paper. A complete version of the paper is available upon request.
} 
each case, $Z_{I P}$ is solved with maximum number of 1000 iterations. The improvement counter is given 25 . Average time consumed in normal case and failure case is up to $3800(\mathrm{sec})$ and $420(\mathrm{sec})$, respectively. The error gap defined by (UB-LB)/LB* $100 \%$ is calculated near $45 \%$ in all cases. Given 1 and 3 Erlangs for voice and data OD pair, respectively, Fig. 1 illustrates the cost analysis as a function of number of mobile stations. No matter what scenario is considered, it takes around $30 \%$ expenses to guarantee survivability. Unavoidably, increasing cost is needed to considering survivable network planning.

The effect of traffic intensity on planning cost is also compared with respect to a number of offered Erlangs. Fig. 2 compares the cost analysis as a function of data intensity with given constant voice intensity 1.0 Erlangs, while cost analysis as a function of voice intensity with given constant data intensity 3 Erlangs is depicted in Fig. 3. Those results indicate traffic intensity affects significantly cost expenses in overall system planning; data traffic is more costly than voice traffic especially. In average, the proposed algorithm is shown to be more effective and achieves up to $25 \%$ reduction on the total deployment cost over a simple algorithm.

\section{CONCLUSION}

Due to the ever-growing user demands and the advances of technology, CDMA has been received increasing attention. However, it still remains a challenge for system planners, managers and administrators, to plan and manage such complex systems in an efficient and effective way. In this paper, the integrated model for survivable CDMA network planning is proposed. We express our achievements in terms of formulation and performance. This research proposes a generic modeling for CDMA network planning.

\section{REFERENCES}

[1] C. U. Saraydar and A. Yener, "Adaptive cell sectorization for CDMA systems," IEEE J. Select. Areas Commun., vol. 19, pp. 1041-1051, June 2001

[2] C. Y. Lee, H. G. Kang, and T. Park, "A dynamic sectorization of microcells for balanced traffic in CDMA: genetic algorithms approach," IEEE Trans. Veh. Technol., vol. 51, pp. 63-72, Jan. 2002.

[3] W. S. Kim and V. K. Prabhu, "Enhanced capacity in CDMA systems with alternate frequency planning," in Proc. IEEE ICC, pp. 973-978, 1998.

[4] K. Hamidian and J. Payne, "Performance analysis of a CDMA/FDMA cellular communication system with cell splitting," in Proc. IEEE ISCC, pp. 545-550, 1997.

[5] S. Hanly and R. Mathar, "On the optimal base-station density for CDMA cellular networks," IEEE Trans. Commun., vol. 50, pp. 1274-1281, July 2002.

[6] E. Amaldi, A. Capone, and F. Malucelli, "Planning UMTS base station location: optimization models with power control and algorithms," IEEE Trans. Wireless Commun., vol. 2, pp. 939-952, 2003.

[7] Kuo-Chung Chu, Frank Yeong-Sung Lin, and Shih-Hao Lee, "Integrated planning and capacity management of survivable DS-CDMA networks," in Proc. IEEE ICNSC, pp. 1154-1159, 2004

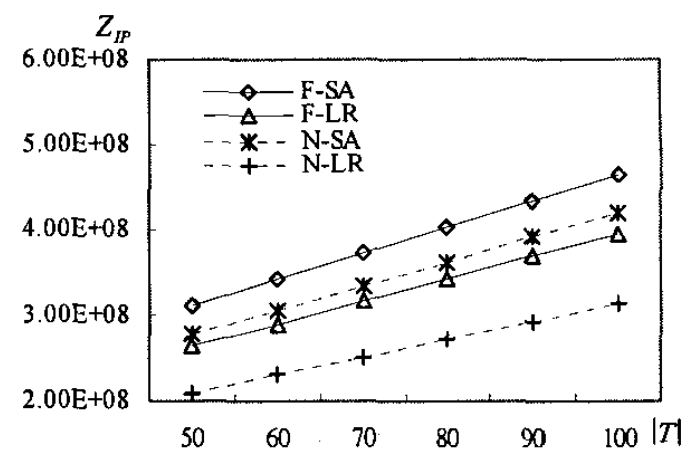

Fig. 1. Planning cost as a function of MS number with respect to algorithms and network states.

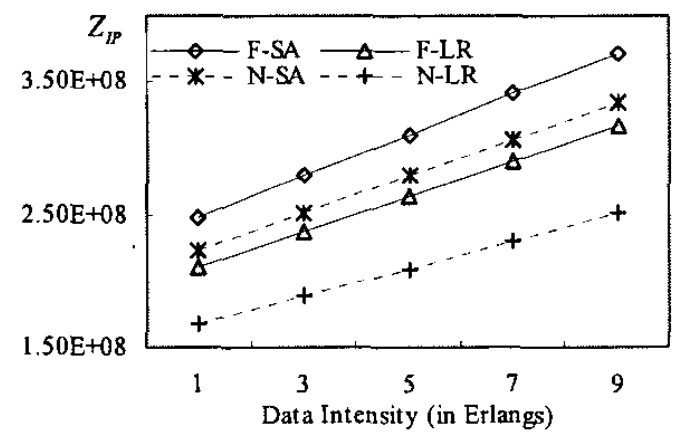

Fig. 2. Planning cost as a function of data traffic intensity with respect to algorithms and network states, given voice intensity 1 Erlangs and $|T|=50$,

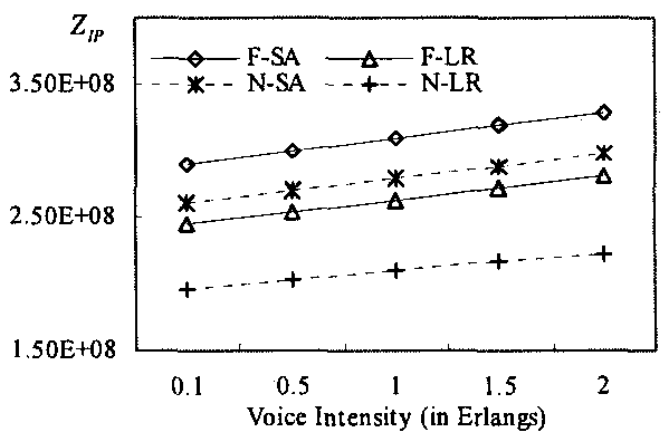

Fig. 3. Planning cost as a function of voice traffic intensity with respect to algorithms and network states, given data intensity 3 Erlangs and $|7|=50$.

[8] Kuo-Chung Chu and Frank Yeong-Sung Lin, "Adaptive load balancing of cellular CDMA systems considering non-uniform traffic distributions," forthcoming, Lecture Notes in Computer Science (Proceedings of HiPC), 2004.

[9] J. S. Kaufman, "Blocking in a shared resource environment," IEEE Trans. Commun., vol. 29, pp. 1474-1481, 1981.

[10] M. L. Fisher, "The Lagrangian Relaxation Method for Solving Integer Programming Problems," Management Science, vol. 27, pp. 1-18, 1981. 